\title{
RELACIÓN ENTRE LOS TIPOS DE FAMILIA Y EL NIVEL DE JUICIO MORAL EN UN GRUPO DE ESTUDIANTES UNIVERSITARIOS
}

\author{
RELATIONSHIP BETWEEN FAMILY TYPES AND MORAL JUDGMENT LEVEL IN \\ UNIVERSITY STUDENTS \\ Ana Delgado V. ${ }^{1}$, Enrioue Ricapa Z. \\ Universidad Nacional Mayor de San Marcos, Perú
}

(RECIBIDO EL 2/07/2010, ACEPTADO EL 5/10/2010)

\begin{abstract}
RESUMEN
Objetivos: a. Conocer la relación entre los tipos de familia y el nivel de juicio moral en un grupo de estudiantes universitarios de la Facultad de Psicología de la UNMSM del décimo ciclo de la especialidad de Psicología Clínica. b. Identificar los tipos de familia del grupo de estudiantes universitarios de la Facultad de Psicología de la UNMSM del décimo ciclo de la especialidad de Psicología Clínica. c. Identificar el nivel de juicio moral de un grupo de estudiantes universitarios de la Facultad de Psicología de la UNMSM del décimo ciclo de la especialidad de Psicología Clínica d. Determinar la relación entre el tipo de familia y el nivel de juicio moral pre convencional de un grupo de estudiantes universitarios de la Facultad de Psicología de la UNMSM del décimo ciclo de la especialidad de Psicología Clínica. e. Determinar la relación entre el tipo de familia y el nivel de juicio moral convencional de un grupo de estudiantes universitarios de la Facultad de Psicología de la UNMSM del décimo ciclo de la especialidad de Psicología Clínica. Diseño: Estudio correlacional. material y métodos: La muestra estuvo contituida por 55 participantes de la Facultad de Psicología de la UNMSM, matriculados en el décimo ciclo de la especialidad de Psicología Clínica. Se utilizó para la recolección de datos la Escala de Evaluación de la Adaptabilidad y Cohesión familiar (FACES III) y el Cuestionario de Reflexión Socio Moral (SROM) de Gibbs y Widaman. Resultados: El 51\% de los universitarios evaluados se encuentran en el nivel convencional de juicio moral. El tipo de familia "caótica dispersa" es la de mayor frecuencia (12) y representa el $22 \%$ de la muestra. En cuanto al nivel de funcionamiento familiar el de mayor frecuencia es el "de rango medio" que representa el $51 \%$ de los participantes. Conclusiones: El nivel de funcionamiento familiar predominante en los evaluados es el "de rango medio. Asimismo, el nivel de juicio moral predominante de la muestra es el convencional. Se encontró una relación estadísticamente significativa entre el nivel de funcionamiento familiar de "rango medio" y el nivel convencional de juicio moral.
\end{abstract}

Palabras clave: Tipos de familia, juicio moral, estudiantes de Psicología del décimo ciclo, especialidad Psicología Clínica.

1 Profesora Principal de la Facultad de Psicología de la UNMSM. E-mail: aedvdt2@yahoo.com 


\begin{abstract}
Objectives: a. To know the relationship between family types and the moral judgement level of tenth semester Clinical Psychology college students at UNMSM. b. Identify the family types of tenth semester Clinical Psychology college students at UNMSM. c. Identify the moral judgement level of tenth semester Clinical Psychology college students at UNMSM. d. To determine the relationship between the family type and the pr.conventional moral judgement level of tenth semester Clinical Psychology college students at UNMSM. e. To determine the relationship between the family type and the conventional moral judgement level of tenth semester Clinical Psychology college students at UNMSM. Design: Correlational study. Materials and methods: The participants were 55 tenth semester Clinical Psychology college students at UNMSM. It was used the Family Adaptability and Cohesion Evaluation Scales (FACES III) and Gibbs and Widam's Sociomoral Reflection Objective Measure (SROM). Results: 51 percent of college students are at the conventional moral judgement level. The chaoti.dipersed family type is the most frequent (12) and represents the $22 \%$ of the sample. Considering the family operating level, the "middle range" is the most frequent and represents the $51 \%$ of participants. Conclusions: The family operating level most frequent is the "middle range" type. Also, the predominant moral judgement is the conventional one. It was found a significative statistical relationship among, family operating "middle range" level and conventional moral judgement level.
\end{abstract}

Keywords: Family types, moral judgement, tenth semester psychology students, clinical psychology major.

\title{
INTRODUCCIÓN
}

Los valores facilitan las relaciones armoniosas entre las personas, repercuten en la sociedad e inciden en el ámbito personal, social, laboral y económico. Una sociedad que proporciona mayor importancia a los valores, tiene una guía que le permite definir su objetivos, prioridades y traducirlos en acciones.

Para Dugui, Macher, Mendoza y Núñez (1995), participar en la edificación de una sociedad con valores sólidos, supone que la conducta de sus líderes actúe en base a ellos para reencauzar el esfuerzo hacia la honestidad y la justicia. No obstante, se lucha contra corriente, pues se está expuesto a modelos de cuestionable calidad moral, que alcanzan injustamente éxito económico o político, mostrando que los conceptos éticos, pueden tomarse a la ligera, donde se otorga preferencia al privilegiado y triunfador, inclusive a aquellos que obtienen sus logros a través de medios ilícitos.

Dicha exposición de antivalores a través de la televisión y otros medios de comunicación, provoca malestar e incapacidad para promover cambio y justicia, atentando contra costumbres y tradiciones directamente en organismos básicos de la sociedad como la familia.

Indicios de deterioro social como la violencia urbana, falta de respeto a la autoridad, crueldad física y verbal contra los niños, conductas autodestructivas, empobrecimiento del lenguaje, embarazos adolescentes, deserción escolar, uso de armas, entre otros, han cambiado la forma en que se representa mentalmente la sociedad. Por tanto, la promoción de valores se ha vuelto una necesidad de ahí la importancia de la intervención en este tema a través de campañas o cruzadas por los valores. 
Para Minuchin y Fishman (1981), la formación de valores es una responsabilidad compartida entre la familia, la sociedad y las personas. La familia es la primera educadora moral del niño e imprime la influencia más perdurable, dado que sus relaciones están revestidas de un carácter emocional especial, que repercute en que los niños se sientan amados y valiosos o bien insignificantes dependiendo de que actúen o no según los preceptos familiares. Los padres transmiten a sus hijos una visión de la vida y las razones por las que es importante actuar conforme a los valores. La combinación amo.autoridad es fundamental. Los niños que tienen una relación estrecha, de comunicación abierta y clara con sus padres, son menos vulnerables a la presión negativa del grupo de pares.

Según Lafosse (1984), la familia es un grupo de personas unidas por lazos de matrimonio, la sangre o la adopción, constituyendo una sola unidad doméstica interactuando y comunicándose entre ellas en sus funciones sociales respectivas de marido y mujer, madre y padre, hijo e hija, hermano y hermana; creando y manteniendo una cultura común.

De acuerdo a Dughi et al. (1995), cualquiera sea el contexto histórico social en que se le examine, la familia debe cumplir cuatro tareas esenciales:

1. Asegurar la satisfacción de las necesidades biológicas del niño y complementar sus inmaduras capacidades de un modo apropiado en cada fase de su desarrollo.

2. Enmarcar, dirigir y canalizar los impulsos del niño con miras a que llegue a ser un individuo integrado, maduro y estable.

3. Enseñarle los roles básicos, así como el valor de las instituciones sociales y los modos de comportarse propios de la sociedad, constituyéndose en el sistema social primario.

4. Transmitir las técnicas adaptativas de la cultura incluido el lenguaje.

Según Dughi et al. la familia puede ser vista como un sistema sociocultural abierto, compuesto por individuos que tienen entre sí lazos de parentesco de tipo consanguíneo o de tipo político. Este sistema es capaz de autorregularse, fijarse objetivos a largo plazo y mantener su equilibrio intercambiando continuamente materia, energía e información con su medio ambiente. Ella mantiene su estabilidad a lo largo del proceso de desarrollo porque es capaz de cambiar constantemente, sin dejar de ser ella misma. Tampoco la composición o el número de sus integrantes es inmutable, sino que se modifican con el tiempo, al igual que sus objetivos, sus reglas y sus procesos de control.

La familia no es un bloque monolítico. Dentro de ella existen subsistemas (el subsistema marital o parental, el subsistema parentofilial y el subsistema fraternal), los cuales son definidos por sus límites. Estos, a su vez, están determinado por reglas que deciden quienes interactúan entre sí y de que manera.

Las familias "adaptativas" o "funcionales" tienen límites bastante definidos y jerarquías netas, y son capaces de establecer relaciones adecuadas con otros subsistemas de su entorno. Con ellas -familia extendida, vecinos, centro laboral, etc.- constituyen redes familiares y sociales de soporte que tal como su nombre lo indica, prestan un valioso apoyo al sistema familiar especialmente cuando éste atraviesa momentos de crisis.

Por el contrario, las familias "inadaptativas" o "disfuncionales" son aquellas en las que los límites o jerarquías se hallan pobremente definidos y dificultan el proceso de separación- 
individuación. En cuanto a sus relaciones con el medio, o se repliegan excesivamente sobre sí mismas, "aglutinando" a sus miembros y trabando su acceso a la autonomía, o se muestran demasiado abiertas, lo que interfiere con la adquisición del sentimiento de pertenencia. Una familia demasiado abierta suele ser incapaz de procesar el exceso de "información" o de mensajes con frecuencia contradictorios provenientes de los otros sistemas. Dicha sobrecarga puede conducirla a la desorganización.

Desde la perspectiva sistémica el funcionamiento de la familia no puede ser entendido plenamente por el simple proceso de comprensión de cada una de sus partes. La estructura y organización de la familia son factores de suma importancia en la conducta de sus miembros y los patrones de interacción del sistema familiar condicionan el comportamiento de cada uno sus integrantes.

De acuerdo a Minuchin y Fishman (1981), estructuralmente la familia es un conjunto de demandas funcionales que organizan los modos en que interactúan sus miembros y contemplan aspectos de su organización tales como subsistemas, roles y jerarquías.

Desde el punto de vista funcional, se enfocan los procesos y patrones de interacción a través de los cuales la familia cumple con sus funciones afectivas e instrumentales.

De acuerdo a Olson, Russell y Sprenkle (1983), el Modelo Circumplejo plantea tres dimensiones centrales del comportamiento familiar: Cohesión, adaptabilidad y comunicación. Estas dimensiones permiten establecer 16 categorías de familias, las cuales según el grado de funcionalidad de su interacción se ubican en tres rangos:

- Familias balanceadas: Las cuales podrían establecer un equilibrio en los niveles de apego y de flexibilidad para el cambio, gracias a su adecuado proceso de comunicación.

- Familias de rango medio: Las cuales serían extremas en alguna de las dimensiones de cohesión o adaptabilidad, pero balanceadas en la otra.

- Familias extremas: Las cuales tendrían muy altos o muy bajos niveles de apego y de flexibilidad.

Para Olson et al. (1983), la cohesión familiar se define como el vínculo emocional que los miembros de la familia tienen entre sí; evalúa el grado en que los miembros de la familia están separados o conectados a ella. Dentro del Modelo Circumplejo, los conceptos específicos para medir y diagnosticar la dimensión de cohesión son la vinculación emocional, los límites, las coaliciones, el tiempo, el espacio, los amigos, la toma de decisiones, los intereses y la recreación. Existen además cuatro niveles de cohesión familiar, que van desde la cohesión extremadamente baja a la cohesión extremadamente alta. Estos se expresan en los siguientes rangos: dispersa, separada, conectada y aglutinada. Los niveles moderados o balanceados, son los de las familias separadas y conectadas.

De acuerdo a estos autores, la adaptabilidad (cambio) familiar tiene que ver con la medida en que el sistema familiar es flexible y capaz de cambiar. Se define como la habilidad de un sistema marital o familiar para cambiar su estructura de poder, las relaciones de roles y las reglas de las relaciones, en respuesta al estrés situacional y propio del desarrollo. Los conceptos específicos para diagnosticar y medir la dimensión de adaptabilidad son el poder en la familia (asertividad, control, disciplina), estilo de negociación, relaciones de 
roles y reglas de las relaciones. Existen también cuatro niveles de adaptabilidad familiar, que van desde la adaptabilidad extremadamente baja a la extremadamente alta, esto es: rígida, estructurada, flexible y caótica. Los niveles balanceados o moderados son los de las familias flexibles y estructuradas.

Asimismo, Olson et al. (1983) definen la comunicación familiar como un proceso interactivo donde la comunicación es siempre una acción conjunta bajo un contexto donde las habilidades positivas, tales como la empatía, la escucha reflexiva y comentarios de apoyo, permiten a las familias compartir entre sí necesidades y preferencias cambiantes en relación con la cohesión y adaptabilidad. Las habilidades negativas, tales como los dobles mensajes, los dobles vínculos y críticas disminuyen la habilidad para compartir los sentimientos y restringen, por lo tanto, la movilidad de la familia en las otras dimensiones.

A partir del Modelo Circumplejo, los niveles centrales (cohesión separada y conectada) son los más viables para el funcionamiento de la familia, considerándose problemáticos los niveles extremos (cohesión dispersa y aglutinada). Los niveles centrales de adaptabilidad (estructurada y flexible) son más favorables para el funcionamiento del matrimonio y la familia. Los extremos (rígida y caótica) son los más problemáticos. La mayoría de parejas y familias que buscan tratamiento se encuentran en uno de estos niveles extremos.

De acuerdo a Polain.Lorente y Martínez (1998), en la familia, la dinámica de interacción entre los miembros es fundamental en la formación de la personalidad de sus nuevos miembros. Ello pues, condicionará una accesibilidad mayor o menor a la autorrealización como individuo. Para Olson et al. (1983), cuando en la dinámica familiar existe equilibrio entre la morfogénesis (cambio) y la morfostasis (estabilidad), suele haber un estilo de comunicación mutuamente asertivo, éxito en las negociaciones, un liderazgo más o menos igualitario, circuitos de retroalimentación positivos, roles y reglas compartidos, siendo aquellos más explícitos que implícitos. Por contraposición, los sistemas familiares disfuncionales se sitúan en los extremos de esta dimensión y suelen tener más dificultades a lo largo de su ciclo vital.

Dugui et al. (1995) consideran que después de la familia, la escuela tiene el rol de educadora moral. La formación y educación no pueden separarse de un sistema de valores. En ocasiones, éste no es explícito, sino que está oculto en el proceso educativo. Por tanto, se quiera o no, se trabaje conscientemente o no, los valores se inculcan y transmiten de una u otra forma. Hacerlo explícitamente implica una coordinación de esfuerzos y una preparación en educación moral que podría permitir a las instituciones y familias actuar en conjunto para no caer en contradicciones y conflictos y para que se respeten las opciones individuales, sin menoscabo de la transmisión de valores universales positivos deseables en todo individuo y sociedad.

Según Shaffer (2000), la moralidad es el conjunto de principios o ideales que ayudan al individuo a distinguir el bien del mal, a actuar de acuerdo con esta distinción y a sentirse orgulloso de la conducta virtuosa y culpable o avergonzado de la conducta que viola sus normas. Señala que la moralidad tiene tres componentes:

El afecto moral: Que es el componente emocional de la moralidad, incluye sentimientos como culpa, vergüenza y orgullo por la conducta ética. 
El razonamiento moral: Que es el componente cognoscitivo de la moralidad, es el pensamiento que demuestran las personas cuando deciden si diversos actos son buenos o malos.

El comportamiento moral: Que es el componente conductual de la moralidad y vienen a ser las acciones congruentes de los individuos hacia las normas morales personales en situaciones en las que surge la tentación de violar dichas normas.

Los teóricos más importantes que han investigado el aspecto cognoscitivo del desarrollo moral, Piaget (1987), Selman (1971) y Kohlberg (1992), coinciden en que los niños sólo pueden emitir juicios morales sólidos hasta alcanzar un nivel de madurez cognoscitivo que les permite ver los hechos desde el punto de vista de otra persona, así como considerar la intencionalidad de las acciones.

Según Rockeach (1973), los valores son la base para la comprensión de actitudes, son fuente de motivación e influyen en la percepción de las creencias (elementos sociales no modificables). Para el grupo social con el que se interactúa, los valores son percibidos como conductas o expresión de sentimientos.

Así también, un valor es la representación del juicio moral, es decir, aquel razonamiento que distingue situaciones en donde actuar según lo que está bien o está mal. Así, por ejemplo, la "responsabilidad" (entendida como el cumplimiento de una tarea), si se actúa de acuerdo a ella, es decir, congruentemente a lo reconocido como bueno por el entorno, entonces el medio reforzará dicha conducta y dicha respuesta, también repercutirá emocionalmente en la persona, haciéndola sentirse orgullosa de sí misma.

Aunque se hace referencia a la teoría de Kohlberg (1992) como "teoría del desarrollo moral", es más propiamente una descripción del desarrollo del juicio moral. Para muchas personas la moralidad son los valores que se han ido adquiriendo en el entorno social y que sobre esos valores que se tienen se actúa en la experiencia diaria.

Kohlberg (1992) y Kohlberg, Power y Higgins (1998) plantean, desde un enfoque sociocognitivo, que el juicio moral es un tipo de razonamiento organizado en estadios morales, según el cual, para alcanzar un determinado estadio es necesario haber accedido a un estadio lógico (o cognitivo) de igual nivel o de nivel superior. Un individuo puede estar en un estadio lógico más elevado que el moral, pero no a la inversa, es decir, un individuo no puede tener un nivel de juicio moral mayor que su nivel de pensamiento operacional, ya sea concreto o formal.

También precisa que dicha maduración cognitiva es una condición necesaria pero no suficiente para la madurez del juicio moral, indicando que existen otros elementos que influyen en este desarrollo, como por ejemplo la interacción existente entre el niño y su ambiente físico y social. La conducta no sólo se ve afectada por el razonamiento, sino por el modelamiento y la internalización. Su investigación ha demostrado que el concepto de justicia, sobre el que se asienta toda la estructura esencial de la moralidad, cambia y se desarrolla con el tiempo, a medida que se interacciona con el entorno.

Según Kohlberg et al. (1998), el sujeto va construyendo, a lo largo de su vida, niveles cualitativamente diferentes, de juicio o razonamiento moral (estadios evolutivos), los cuales son fruto de la interacción entre tendencias del organismo y las estructuras del 
medio exterior y no mero reflejo. Estos niveles, que implica una relación diferente entre el yo y las reglas y expectativas morales de la sociedad, son los siguientes:

Nivel I. Preconvencional: Para Kohlberg et al. (1998), en este nivel las normas sociales no son comprendidas como tales, los sujetos poseen una perspectiva individual por lo que priman los intereses concretos y el deseo de evitar riesgos, por ello no se adecuan a las normas o sólo se adecuan por miedo al castigo o por interés.

Es el nivel de la mayoría de los niños menores de 9 años, de algunos adolescentes y de muchos delincuentes. Se enfoca la cuestión moral desde los intereses concretos de los individuos implicados y en función de las consecuencias inmediatas de sus actos: Evitación de castigos y defensa de los propios intereses. Las reglas y expectativas son aún externas al yo. Todavía no se comprenden y tampoco se defienden. A nivel cognitivo, son personas que mantienen un enfoque muy concreto. Su razonamiento moral se basa en el nivel preoperatorio o de las operaciones concretas del desarrollo cognitivo.

Nivel II. Convencional: De acuerdo a Kohlberg et al. (1998), en este nivel existe la conciencia de ser miembro de un grupo social, por lo que se da una identificación del sujeto con las normas de su grupo y reconoce que la sociedad espera que actúe de acuerdo a las normas establecidas. El responder a las expectativas sociales es un valor en sí mismo sin que haya consecuencias a favor propio.

Es el nivel de la mayoría de los adolescentes y adultos. El individuo ya se orienta en función de las expectativas de los demás o del mantenimiento del sistema social. El término "convencional" implica someterse a las reglas, las expectativas y las convenciones sociales, y a la autoridad y defenderlas, porque son reglas, expectativas y convenciones de la sociedad. El yo se identifica, por tanto, con las reglas y expectativas de los demás. Las personas que adoptan una perspectiva convencional consideran las cuestiones más abstractas de lo que su sociedad esperaría de ellos. Están empleando el razonamiento moral que se basa por lo menos en las primeras operaciones formales.

Nivel III. Post convencional: Según Kohlberg et al. (1998), en este nivel el sujeto entiende y se puede identificar con normas sociales, pero va más allá de ellas. Se orienta por valores que responden a principios, que comprende y elige personalmente y que coinciden con los principios éticos, universales.

Este nivel es alcanzado únicamente por una minoría de adultos y sólo a partir de los 2.23 años. Se construyen principios morales autónomos que permitirían llegar a una sociedad ideal. Se ve más allá de las normas y leyes dadas por la sociedad. Se comprenden y aceptan básicamente las reglas de la sociedad, pero dicha aceptación se basa precisamente en la formulación y aceptación de los principios morales de carácter general que están debajo de esas reglas. Estos principios entran en algunas ocasiones en conflicto con las reglas de la sociedad, en cuyo caso el sujeto que está en este nivel juzga por el principio más que por la convención. Hay una diferencia clara entre el yo y las reglas y expectativas sociales. Defiende sus valores en función de los principios escogidos en ella sin precisión de la autoridad o las reglas. Estas personas piensan en las categorías puramente formales de lo que sería la mejor solución dados estos principios morales. Emplean un razonamiento basado en operaciones formales avanzadas o consolidadas. 
Desde estos postulados desarrolla seis estadios de desarrollo moral, cada uno de los cuales proporciona un sistema de razonamiento moral más complejo y, por tanto, un concepto más adecuado de lo que es justo y bueno. Estas etapas son universales, aunque los factores del entorno influyen en el ritmo de crecimiento y el nivel de desarrollo conseguido.

Por otro lado, se espera que las personas tengan características predominantes de un estadio, y características secundarias del estadio anterior. Además, explica que todos los niños pasan los mismos estadios de desarrollo en su juicio moral; algunos lo logran más rápidamente y otros llegan a niveles superiores, dependiendo del ambiente en el cual se desarrollan.

De acuerdo a Kohlberg (1992), se tienen las siguientes definiciones para los estadios:

Estadio 1: Moral heterónoma. El absolutismo y la orientación al castigo (hasta los 7 u 8 años de edad)

Tiene las siguientes características: Ingenuo realismo moral que lleva a comprender el significado moral de una situación como una cualidad física inherente a la propia acción, aplicación absoluta o literal de etiquetas o reglas sin requerir justificación alguna, hay una marcada orientación al castigo e incapacidad para distinguir distintas perspectivas sociales en los dilemas morales planteados.

Se presenta unilateralidad y ausencia de coordinaciones recíprocas, es decir, incapacidad para equilibrar mentalmente las distintas posiciones sociales. La justicia se define en función de las diferencias de poder y estatus. Existe dificultad para reconocer y describir conflictos entre valores en situaciones reales. Lo correcto es evitar violar las reglas sustentadas por el castigo y la obediencia misma, así como evitar el daño físico a personas y bienes. Las razones para hacer lo correcto son evitar el castigo y el temor al poder superior de las autoridades.

Esta etapa tiene un carácter egocéntrico. No considera los intereses de los demás y no relaciona dos puntos de vista distintos. Las acciones son consideradas físicamente y no en términos de los intereses psicológicos de otros. Existe confusión de la perspectiva de la autoridad con la propia.

Una condición necesaria para superar este estadio es la utilización de la reversibilidad lógica en la comprensión de la realidad física y social, lo que coincide con el pensamiento operatorio concreto descrito por Piaget.

\section{Estadio 2: El individualismo instrumental, propósito e intercambio}

Se caracteriza porque lo correcto es seguir las reglas cuando responde al interés inmediato de uno. Se actúa para satisfacer los propios intereses y necesidades, permitiendo que otros hagan lo mismo. Lo correcto es lo justo, un intercambio equitativo, un trato o un acuerdo. Las razones para hacer lo correcto son servir a los propios intereses y necesidades, en un mundo donde se debe reconocer que otras personas también tienen sus intereses. 
La perspectiva de esta etapa es individualista concreta. Se tiene conciencia de que todos persiguen sus propios intereses y de que estos entran en conflicto, de modo que lo correcto es relativo. Se supera el ingenuo realismo anterior y se descubre que existen conflictos de intereses y que las soluciones unilaterales, basadas exclusivamente en la obediencia y el castigo, son inadecuadas. Las acciones morales exigen reciprocidad. Se coordinan los intereses en conflicto tratándolos de forma estrictamente igual o a través del intercambio (estructura individualista instrumental).

El concepto de justicia se entiende como intercambio de favores: "Haz a los demás lo que te hacen a ti o esperas que te hagan". Reciprocidad simple y concreta (comienzo de la reciprocidad moral). Existe una orientación hedonist.instrumental que le lleva a garantizar al máximo la satisfacción de necesidades y deseos, reduciendo al mínimo las consecuencias negativas para sí mismo.

Estadio 3: La moral de las relaciones, expectativas interpersonales mutuas y conformidad interpersonal (a partir de los 12/13 años)

Tiene las siguientes características: Lo correcto es estar a la altura de lo que espera la gente, que está cerca de uno, o lo que la gente espera en general de otros, en el rol de hijo, hermano, amigo, etc. "Ser bueno" es importante y significa tener buenos motivos, demostrar preocupación por los otros. También significa mantener relaciones mutuas, como confianza, lealtad, respeto y gratitud.

Las razones para hacer lo correcto son la necesidad de ser buena persona a los propios ojos y a los demás. Es preocuparse por los otros, creer en la regla de oro. Significa además desear mantener las reglas y la autoridad que apoyan la conducta estereotípicamente buena.

La perspectiva de esta etapa del individuo está en función de las relaciones con otros individuos. Existe conciencia de sentimientos, acuerdos y expectativas compartidas, que tienen primacía sobre los intereses individuales. Relaciona los puntos de vista mediante la Regla de Oro concreta, poniendo a uno en el lugar del otro individuo. Aún no considera la perspectiva del sistema generalizado.

El individuo es capaz de adoptar la perspectiva de una tercera persona. Se supera el individualismo instrumental y se construyen un conjunto de normas o expectativas morales compartidas que se espera que cumplan todos. Las normas o expectativas morales son la base para establecer relaciones de confianza mutua que trascienden los intereses particulares.

Hay una orientación a los sentimientos y las relaciones interpersonales, se presenta una preocupación especial por la confianza y la aprobación social. Las obligaciones morales se basan en lo que esperan los demás (perspectiva convencional) y se anteponen las expectativas y sentimientos de los demás a los propios intereses (es importante ser bueno). El concepto de justicia se entiende de la siguiente manera: "Haz a los demás lo que te gustaría que te hicieran a ti si estuvieras en su lugar". Hay mayor nivel de reciprocidad. 
Este estadio caracteriza al razonamiento de la mayoría de los adolescentes y adultos tanto en situaciones hipotéticas como reales, observándose gran consistencia. El pensamiento formal que comienza a desarrollarse en esta edad proporciona una herramienta intelectual que permite gran independencia del contenido concreto sobre el que se aplica. Este tipo de pensamiento es una condición necesaria, aunque no suficiente, para el estadio 3 de razonamiento moral.

\section{Estadio 4: La moral de la coherencia y el sistema social. Sistema social y conciencia}

Presenta las siguientes características: Lo correcto es cumplir con los deberes efectivos que uno ha aceptado. Se deben sostener las leyes salvo en casos extremos en que entran en conflicto con otras obligaciones sociales fijas. Lo correcto también es contribuir a la sociedad, el grupo o la institución.

Las razones para hacer lo correcto es mantener la institución en funcionamiento, evitar el colapso del sistema ("si todos lo hicieran"), o el imperativo de la conciencia de cumplir las obligaciones definidas (que se confunde fácilmente con la creencia en las reglas y la autoridad de la etapa 3).

La perspectiva social de esta etapa es la diferenciación de los puntos de vista societarios de acuerdo a los motivos interpersonales. Toma el punto de vista del sistema que define roles y normas, y considera las relaciones individuales en términos del lugar en el sistema.

Este estadio no se da antes de los 16 años y su frecuencia en la población adulta es menor que el estadio 3. La integración de las expectativas interpersonales y las normas compartidas se da dentro de un sistema más amplio y generalizado (la perspectiva de un miembro de la sociedad) que se concreta en un conjunto consistente de códigos y procedimientos que se aplican imparcialmente a todos sus miembros. Se juzgan, por tanto, las situaciones particulares según su función en la sociedad como un todo. Hay una acentuada preocupación por la coherencia y la imparcialidad, por seguir lo que indica la propia conciencia y mantener el respeto hacia uno mismo. La concepción de la justicia está basada en la consideración de los deberes en relación a sus derechos recíprocos.

\section{Estadio 5: La moral de los derechos humanos. Contrato o utilidad social y derechos individuales}

Se caracteriza porque lo correcto es tener conciencia de que la gente posee una variedad de valores y opiniones; que la mayoría de los valores y reglas son relativos al propio grupo. Pero esas reglas, relativas en general, deben ser sostenidas en el interés de la imparcialidad y porque son el contrato social. Sin embargo, algunos valores y derechos no relativos, como vida y libertad, deben ser sostenidos en toda sociedad y con independencia de la opinión de la mayoría.

Las razones para hacer lo correcto son el sentido de obligación hacia la ley debido al contrato social de hacer y respetar las leyes para el bienestar de todos y para la protección del derecho de toda la gente. Un sentido de compromiso contractual, contraído libremente, con la familia, la amistad, la confianza y la obligación de trabajar. Existe la preocupación 
de que las leyes y los deberes se basen en el cálculo racional de la utilidad general ("el mayor bien para el mayor número").

La perspectiva social de esta etapa es la de un individuo racional conciente de los valores y los derechos propios a las vinculaciones y los contratos sociales. Integra perspectivas mediante mecanismos formales de acuerdo, contrato, imparcialidad objetiva y debido proceso. Además, considera los puntos de vista moral y legal; reconoce que a veces están en conflicto y le resulta difícil integrarlos.

La acción concreta tiende a definirse en términos de derechos legales, sobre los que está de acuerdo la sociedad en su conjunto. Hay un énfasis en el punto de vista legal, pero las leyes no son eternas, sino instrumentos flexibles para profundizar en los valores morales, que pueden y deben cambiarse para mejorarlas.

El contrato social supone la participación voluntaria en un sistema social aceptado, porque es mejor para uno mismo y los demás que su carencia. Se concibe el sistema social ideal como un contrato libremente asumido por cada individuo para garantizar los derechos y el bienestar de todos sus miembros.

Se va adoptando una perspectiva autónoma, que va más allá de la sociedad, basada en principios que podrían ser aceptados por cualquier agente moral racional para construir una sociedad ideal. Se establece una jerarquía de prioridades entre los derechos, en función de la cual se toman decisiones cuando existen conflictos entre varios de ellos. Hay una especial preocupación por proteger los derechos de las minorías y de las personas en situación de desventaja y por el bienestar social.

\section{Estadio 6: Principios éticos universales}

Tiene las siguientes características: Lo correcto es seguir los principios éticos elegidos por uno mismo. Las leyes o los acuerdos sociales particulares suelen ser válidos porque se basan en tales principios (actúa de acuerdo al principio). Los principios, son principios universales de justicia, la igualdad de los derechos humanos y el respeto por la dignidad de los seres humanos como personas individuales.

Las razones para hacer lo correcto son la creencia como persona racional en la validez de los principios morales universales y en el sentido de compromiso personal con ellos. La perspectiva social de esta etapa parte de un punto vista moral del cual derivan los arreglos sociales. La perspectiva es que cualquier individuo racional reconoce la naturaleza de la moralidad o el hecho de que las personas son fines en sí mismas y deben ser tratadas como tal.

Son principios morales abstractos que trascienden las leyes, como la igualdad de los seres humanos y el respeto a la dignidad de cada persona, pero no son normas concretas.

Aparece una forma abstracta de considerar las perspectivas de todas las partes y de tratar de organizarlas con principios generales.

El estadio 5 es el más disponible y por tanto el más común. El 6 representa filosóficamente una posición más adecuada, pero es más rara entre los que no son filósofos. 
Para Barba y Romo (2005), los estudiantes universitarios poseen un nivel de desarrollo moral predominantemente convencional asociado también por el tipo de institución donde estudian, siendo la universidad pública la que posee mayor avance moral que una privada.

Según Polain.Lorente y Martínez (1998), la familia es un ambiente importante de desarrollo, donde el diálogo y la interacción en cuestiones éticas, llevan a los niños a pensar por sí solos y no sólo a repetir respuestas correctas o incorrectas. En el modelamiento, propiciado dentro de la familia en la exposición de su dinámica, es posible que los infantes expuestos de manera consistente a padres generosos y altruistas, respetuosos y cuidadosos con los demás, tiendan a preocuparse más por los derechos y sentimientos de otros. En un inicio, los padres dirigen la conducta moral de los pequeños mediante instrucción directa, supervisión, recompensas, castigos y corrección; pero llega un momento en que los niños internalizan dichas guías y adoptan los estándares externos como propios. De ahí la importancia de darles explicaciones y razones al corregirlos en sus actos, esperando de ellos que se comporten de una manera moral aunque nadie los observe.

Lo anterior evidentemente plantea cuestiones centrales, por lo cual el problema de esta investigación puede ser planteado en los siguientes términos: ¿Cuál es la relación entre los tipos de familia y el nivel de juicio moral en un grupo estudiantes universitarios de la Facultad de Psicología de la UNMSM del décimo ciclo de la especialidad en Psicología Clínica?

\section{Objetivos}

1. Conocer la relación entre los tipos de familia y el nivel de juicio moral en un grupo de estudiantes universitarios de la Facultad de Psicología de la UNMSM del décimo ciclo de la Especialidad en Psicología Clínica.

2. Identificar el nivel de juicio moral de un grupo de estudiantes universitarios de la Facultad de Psicología de la UNMSM del décimo ciclo de la especialidad en Psicología Clínica.

3. Identificar los tipos de familia del grupo de estudiantes universitarios de la Facultad de Psicología de la UNMSM del décimo ciclo de la especialidad en Psicología Clínica.

4. Determinar la relación entre el tipo de familia y el nivel de juicio moral pre convencional de un grupo de estudiantes universitarios de la Facultad de Psicología de la UNMSM del décimo ciclo de la especialidad en Psicología Clínica.

5. Determinar la relación entre el tipo de familia y el nivel de juicio moral convencional de un grupo de estudiantes universitarios de la Facultad de Psicología de la UNMSM del décimo ciclo de la especialidad en Psicología Clínica.

\section{MÉTODO}

En el presente estudio se ha utilizado un diseño de carácter correlacional. De acuerdo a Sánchez y Reyes (2006), este diseño se orienta a la determinación del grado de relación 
existente entre dos o más variables de interés en una misma muestra de sujetos. Así, en la presente investigación, la muestra fue un grupo de estudiantes universitarios de la Facultad de Psicología de la UNMSM del décimo ciclo de la especialidad en Psicología Clínica, a quienes se les aplicó el Cuestionario de Reflexión Socio Moral (SROM) y la Escala de Evaluación de la Adaptabilidad y Cohesión Familiar (FACES III), para obtener respectivamente, puntajes que permitieran conocer el Nivel de Juicio Moral y el Tipo de Familia en dicha muestra. Finalmente, a través del análisis estadístico de estos resultados se obtuvo un coeficiente de correlación, que permitió determinar la relación existente entre estas dos variables.

La población objetivo fue un total de 64 estudiantes de la Facultad de Psicología de la UNMSM, matriculados en el semestre 2004-II, correspondiente al décimo ciclo de la especialidad en Psicología Clínica.

El universo de investigación estuvo conformado por los alumnos matriculados en tercer año de secundaria de Lima Metropolitana. Participaron 679 estudiantes. El tamaño de la muestra se determinó utilizando la fórmula propuesta por Snedecor y Cochran (1981), obteniéndose una muestra de 55 participantes. La selección de los participantes se llevó a cabo utilizando el muestreo aleatorio o al azar. Según Sánchez y Reyes (2006), dentro de los tipos de muestreo probabilístico, el muestreo aleatorio o al azar supone que los miembros de una población tienen iguales posibilidades de pertenecer a la muestra. Se utilizó el listado alfabético de alumnos matriculados proporcionado por la Oficina de Matrícula de la Facultad de Psicología de la UNMSM, para la determinación de los participantes.

En esta investigación se utilizó la Escala de Evaluación de la Adaptabilidad y Cohesión Familiar (FACES III) (Olson, 1985), con la finalidad de identificar el tipo de familia, según el Modelo Circumplejo y el Cuestionario de Reflexión Socio Moral (SROM) de Gibbs y Widaman, con el objetivo de establecer el nivel y etapa de desarrollo del juicio moral de los participantes del estudio.

Se elaboraron tablas de frecuencias, porcentajes y gráficos para presentar los resultados concernientes a los tipos de familia, niveles de funcionamiento familiar, niveles de juicio moral y estadios del juicio moral. Se utilizó la prueba Chi Cuadrado $\left(\mathrm{X}^{2}\right)$ para establecer la asociación entre los tipos de familia y el nivel de juicio moral pre convencional y convencional. Los datos fueron procesados utilizando el programa estadístico SPSS.

\section{RESULTADOS}

Al analizar la distribución de frecuencias y porcentajes de los tipos de familia en la muestra total de estudiantes universitarios de la Facultad de Psicología de la UNMSM (ver Tabla 1 y Gráfico 1), se encuentra que el tipo de familia "caótica dispersa" es la de mayor frecuencia (12) y representa el $22 \%$ de la muestra. Esta se sitúa dentro del nivel de funcionamiento familiar "extremo" (Olson, Russell y Sprenkle, 1983), presentando dificultades en ambas dimensiones, caracterizándose por un liderazgo limitado o ineficaz, disciplinas muy poco severas, decisiones parentales impulsivas, falta de claridad en las funciones, frecuentes cambios en las reglas, extrema separación 
emocional, correspondencia afectiva infrecuente, falta de cercanía paterno filial, falta de lealtad familiar, necesidad y preferencia por espacios separados, toma de decisiones independientes, intereses desiguales y focalizados fuera de la familia.

El tipo de familia "flexible dispersa" en el orden de frecuencias (9), es la siguiente, representando el $16 \%$ de la muestra. Tiene un nivel de funcionamiento familiar "de rango medio" (Olson et al., 1983), es decir presenta dificultades en una sola dimensión, caracterizándose, la saludable, por un liderazgo igualitario y democrático, que permite cambios y acuerdos en las decisiones, aunque la disciplina es algo severa se negocian sus consecuencias y las reglas se hacen cumplir con flexibilidad y pueden cambiar, compartiéndose roles y funciones. La dimensión de funcionamiento problemático, identifica una extrema separación emocional, muy poco involucramiento o interacción entre sus miembros, correspondencia afectiva infrecuente entre sus miembros, falta de cercanía paterno filial, falta de lealtad familiar, necesidad y preferencia por espacios separados, siendo la toma de decisiones independiente, existiendo intereses desiguales y focalizados fuera de la familia.

Seguidamente, el tipo de familia "flexible separada" con una frecuencia de (8) y con un $15 \%$, se enmarca dentro del funcionamiento familiar "balanceado" (Olson et al. 1983), siendo centrada en ambas dimensiones. Se caracteriza por un liderazgo igualitario, que permite cambios, con disciplina algo severa, negociándose sus consecuencias, usualmente es democrática y existe acuerdo en las decisiones, se comparten los roles o funciones, las reglas se cumplen con flexibilidad y algunas cambian. También existe separación emocional, lealtad familiar ocasional, aceptándose el involucramiento, pero prefiriéndose la distancia personal y algunas veces se demuestra la correspondencia personal. Los límites parent.filiales son claros con cierta cercanía entre padres e hijos, se alienta cierta separación emocional, el tiempo individual es importante pero se comparte parte del tiempo, se prefieren los espacios separados compartiendo el espacio familiar, las decisiones se toman individualmente, siendo posible las que se toman en forma grupal, el interés se focaliza fuera de la familia y los amigos personales raramente son compartidos con la familia, los intereses son distintos, la recreación se lleva a cabo más separada que en forma compartida.

Por otro lado, el tipo de familia "caótica aglutinada" y "rígida separada" obtienen la menor frecuencia (1), representando el $2 \%$ de la muestra cada una de ellas. La primera se encuentra dentro del nivel de funcionamiento familiar "extremo" y la segunda dentro del "rango medio". 
Tabla N. ${ }^{\circ}$ 1. Frecuencias y porcentajes de tipos de familia según FACES III.

\begin{tabular}{lcc}
\hline Tipo de Familia (FACES III) & $\mathrm{f}$ & $\%$ \\
\hline Caótica dispersa & 12 & 22 \\
Caótica separada & 7 & 13 \\
Caótica conectada & 6 & 11 \\
Caótica aglutinada & 1 & 2 \\
Flexible dispersa & 9 & 16 \\
Flexible separada & 8 & 15 \\
Estructurada dispersa & 5 & 9 \\
Estructurada separada & 3 & 5 \\
Rígida dispersa & 3 & 5 \\
Rígida separada & 1 & 2 \\
\hline Total & 55 & 100 \\
\hline
\end{tabular}

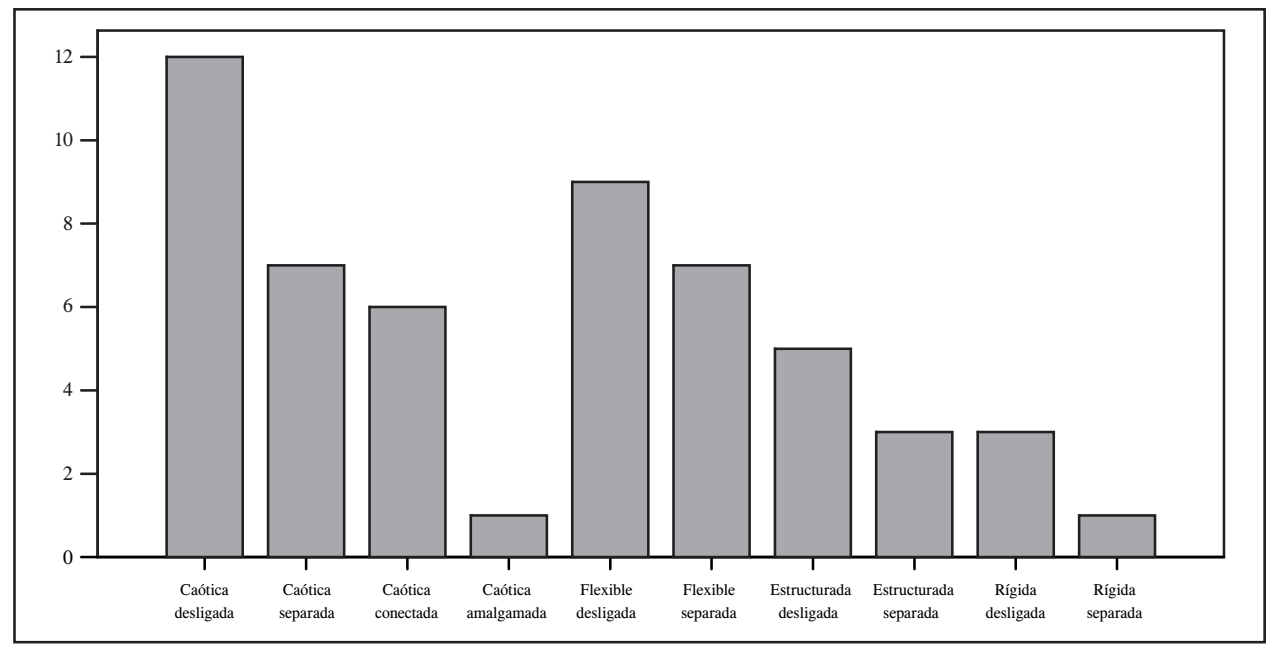

Figura $\mathbf{N}^{\circ}$ 1. Tipos de familia según FACES III.

Con respecto al nivel de funcionamiento familiar (ver Tabla 2 y Gráfico 2), se observa que la mayor frecuencia (28) es la "de rango medio", que representa el $51 \%$ de la muestra, lo cual indica que los sujetos perciben que sus dinámicas familiares presentan dificultad en alguna dimensión de la adaptabilidad o cohesión.

Por otro lado, la menor frecuencia (11), corresponde al nivel de funcionamiento familiar "balanceado" que representa el $20 \%$ de la muestra. 
Tabla N. ${ }^{\circ}$ 2. Frecuencias y porcentajes de niveles de funcionamiento familiar según FACES III.

\begin{tabular}{lcc}
\hline Nivel de funcionamiento familiar & $\mathrm{f}$ & $\%$ \\
\hline Tipo balanceada & 11 & 20 \\
De rango medio & 28 & 51 \\
Extremas & 16 & 29 \\
\hline Total & 55 & 100 \\
\hline
\end{tabular}

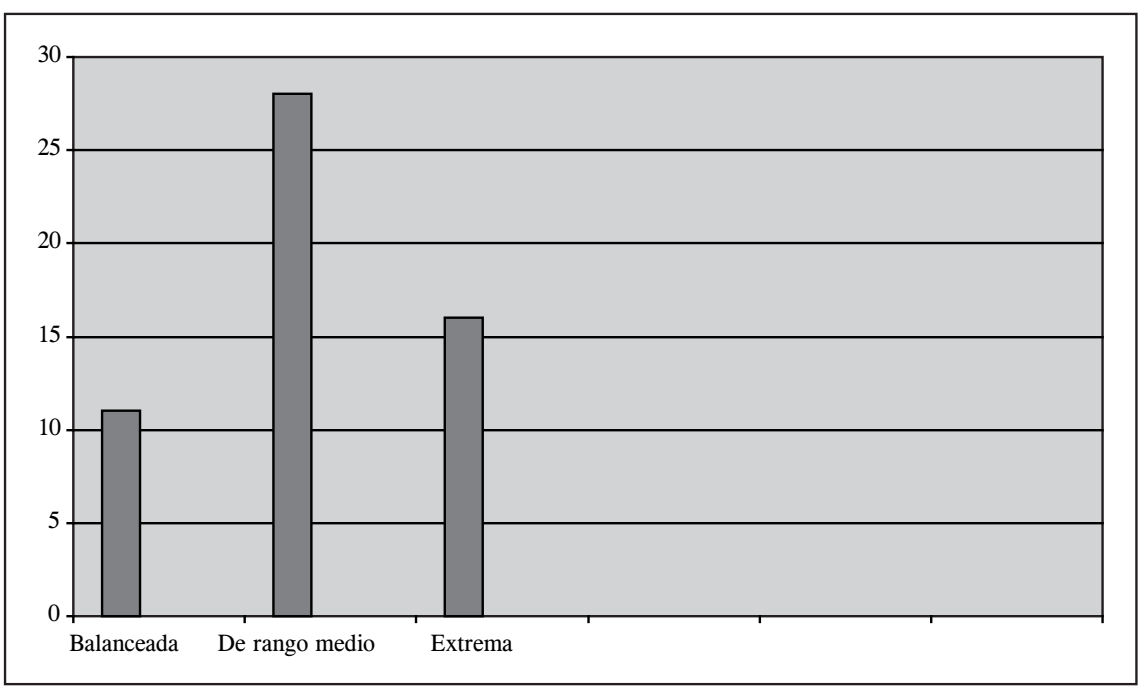

Figura $\mathbf{N} .^{\circ}$ 2. Nivel de funcionamiento familiar.

Asimismo, sobre el nivel de juicio moral (ver Tabla 3 y Gráfico 3), se aprecia que la mayor frecuencia (51) se encuentra dentro del nivel convencional y representa el $51 \%$ de la muestra. Los sujetos que se encuentran dentro este nivel son concientes de ser miembros de un grupo social y se identifican con las normas y reglas, actuando de acuerdo a ellas y considerando un valor en sí mismo dicha conducta, sin esperar una consecuencia a favor propio. De acuerdo a Kohlberg (1992), es el nivel alcanzado que se espera para la edad de la muestra.

La menor frecuencia (4), se encuentra en el nivel pre convencional del juicio moral, que representa el $7 \%$ de la muestra.

Tabla N. ${ }^{\circ}$ 3. Frecuencias y porcentajes del nivel de juicio moral según SROM.

\begin{tabular}{lcc}
\hline Nivel de Juicio Moral (SROM) & $\mathrm{f}$ & $\%$ \\
\hline Pre Convencional & 4 & 7 \\
Convencional & 51 & 93 \\
\hline Total & 55 & 100 \\
\hline
\end{tabular}




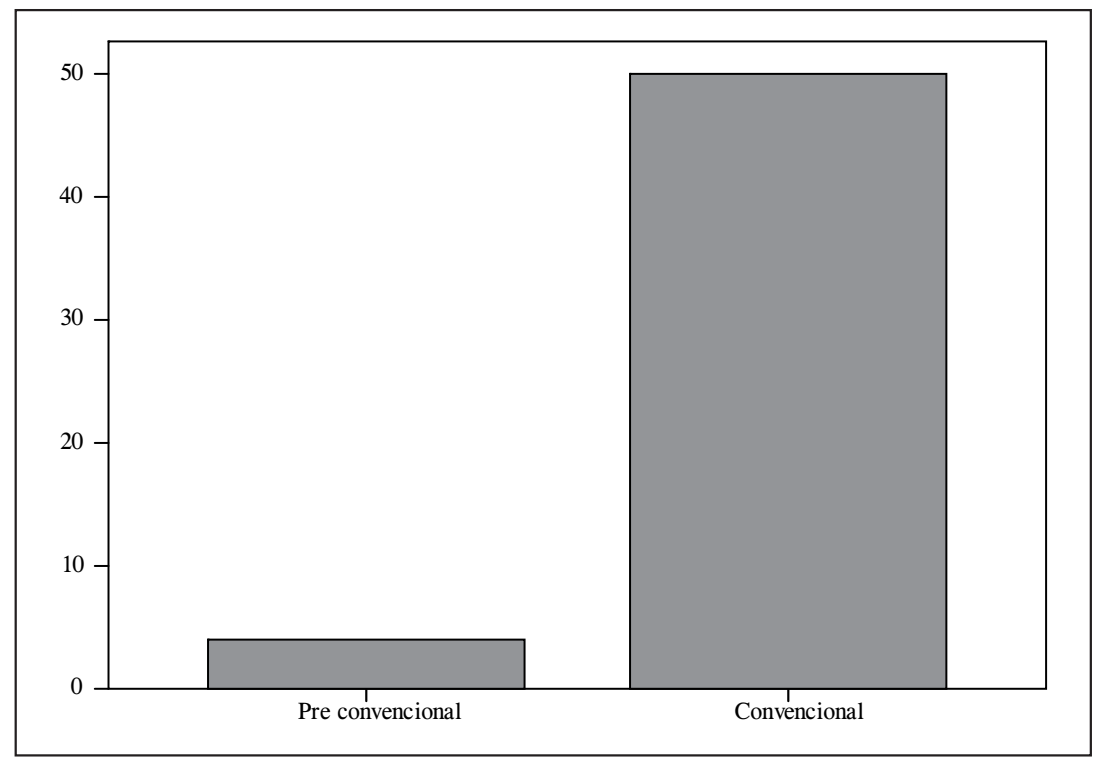

Figura N. ${ }^{\circ}$ 3. Nivel de juicio moral según SROM.

En cuanto a los estadios del desarrollo del juicio moral (ver Tabla 4 y Gráfico 4), se tiene que la mayor frecuencia (38) se concentra en el estadio III, que representa el 69\% de la muestra. Las características de este estadio, según Kohlberg (1992), son el deseo de mantener las reglas y la autoridad que apoyan a la conducta socialmente aceptada. Además, son concientes de que las normas o expectativas morales son la base para establecer relaciones de confianza mutua que trascienden los intereses particulares. Asimismo, este estadio caracteriza el razonamiento de la mayoría de adultos, lo que a su vez se confirma con la edad de la muestra.

La menor frecuencia (4) corresponde al estadio II del juicio moral y representa el $7 \%$ de la muestra.

Tabla N. ${ }^{\circ}$ 4. Frecuencias y porcentajes de estadios del desarrollo del juicio moral según SROM.

\begin{tabular}{lcc}
\hline $\begin{array}{l}\text { Estadios del desarrollo } \\
\text { del juicio moral (SROM) }\end{array}$ & f & $\%$ \\
\hline Estadio II & 4 & 7 \\
Estadio III & 38 & 69 \\
Estadio IV & 13 & 24 \\
\hline Total & 55 & 100 \\
\hline
\end{tabular}




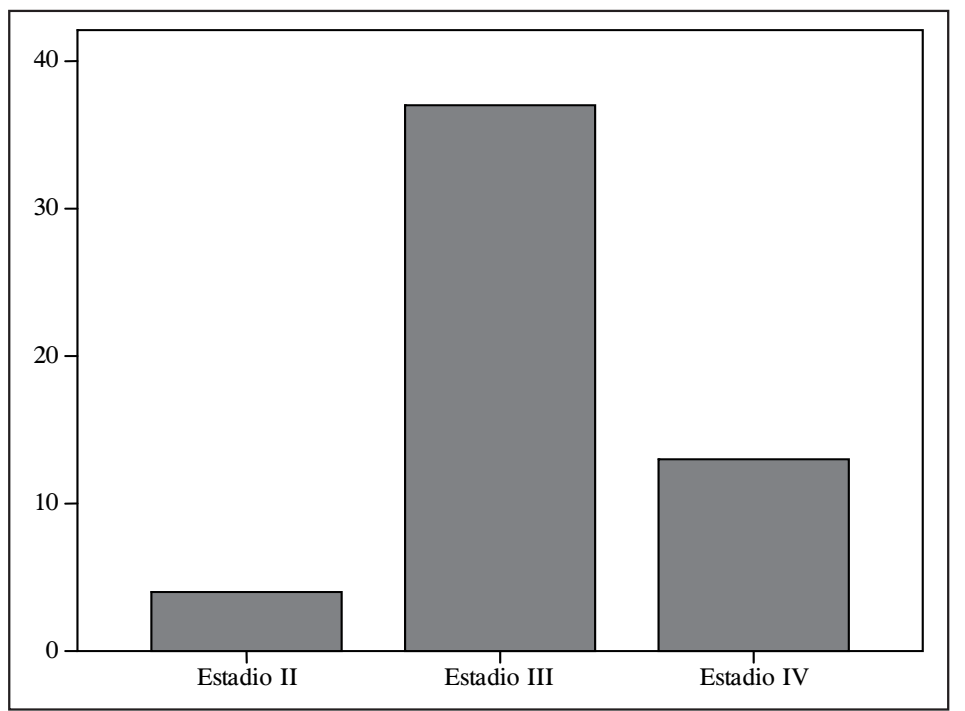

Gráfico N. ${ }^{\circ}$ 4. Estadios del juicio moral según SROM.

Con el fin de analizar la relación entre los tipos de familia y el nivel de juicio moral, se procedió a establecer el grado de dependencia entre estas dos variables, a través de la utilización de la Chi cuadrada, porque a través de este estadístico es posible determinar el grado de relación entre dos variables nominales (Siegel y Castellan, 1995; Alarcón, 2008).

En la Tabla 5 se presentan los resultados acerca del nivel pre convencional con el nivel de funcionamiento familiar, correspondiente al objetivo referido los tipos de familia y el nivel de juicio moral pre convencional en el grupo de estudiantes universitarios de la Facultad de Psicología de la UNMSM y que por simple inspección puede rechazarse algún tipo de asociación válida.

Tabla N. ${ }^{\circ}$ 5. Frecuencias del nivel pre convencional con el nivel de funcionamiento familiar.

\begin{tabular}{lc}
\hline $\begin{array}{c}\text { Nivel de } \\
\text { funcionamiento } \\
\text { familiar }\end{array}$ & $\begin{array}{c}\text { Frecuencia } \\
\text { observada }\end{array}$ \\
\hline Balanceada & 1 \\
Rango medio & 3 \\
\hline Total & 4 \\
\hline
\end{tabular}

Al analizar el nivel convencional con el nivel de funcionamiento familiar (ver Tabla 6), los resultados muestran una Chi cuadrada igual a 6,705, que es un valor estadísticamente 
significativo $(\mathrm{p}<, 05)$, por lo tanto, se puede aceptar lo referido a la existencia de una asociación estadísticamente significativa entre los tipos de familia y el nivel de juicio moral convencional en el grupo de estudiantes universitarios de la Facultad de Psicología de la UNMSM.

Tabla N. ${ }^{\circ}$ 6. Prueba de Chi Cuadrado entre el nivel convencional y el nivel de $\mathrm{f}$ uncionamiento familiar.

\begin{tabular}{lccccc}
\hline $\begin{array}{c}\text { Nivel de } \\
\text { funcionamiento } \\
\text { familiar }\end{array}$ & $\begin{array}{c}\text { Frecuencia } \\
\text { observada }\end{array}$ & $\begin{array}{c}\text { Frecuencia } \\
\text { esperada }\end{array}$ & $\mathrm{X}^{2}$ & $\mathrm{~g} .1$. & $\mathrm{p}$. \\
\hline Balanceada & 10 & 17,0 & 6,705 & 2 &, $035^{*}$ \\
Rango medio & 25 & 17,0 & & & \\
Extrema & 16 & 17,0 & & & \\
\hline Total & 51 & & & & \\
$* \mathrm{p}<, 05$ & & & & &
\end{tabular}

\section{DISCUSIÓN}

El predominio de la moralidad convencional sobre los otros dos niveles en los resultados en la presente investigación indica que la mayoría de los participantes tienden a tomar sus decisiones orientando su razonamiento hacia la importancia del mantenimiento de las relaciones sociales, buscando la aprobación de los demás y demostrando conciencia de que los sentimientos, acuerdos y expectativas compartidas, tienen primacía sobre los intereses individuales (Kohlberg, 1998). También caracterizan su razonamiento, otorgando valor a la confianza, la lealtad, el respeto y la gratitud, que son expresión de su deseo por asegurar las reglas y mostrándose de acuerdo en que debe existir una autoridad que mantenga y apoye la buena conducta, el orden y la moralidad.

Por otro lado, la muestra se ubica mayormente dentro del nivel de funcionamiento familiar "de rango medio" en la cual se encuentra cinco tipos de familia de las ocho teóricas: Flexiblemente dispersa, caóticamente separada, caóticamente conectada, estructuralmente dispersa y rígidamente separada. Todas ellas tienen como denominador común la desviación extrema hacia una de las dimensiones del funcionamiento familiar, pero el balance en la otra; es decir, o bien son extremas en cohesión y balanceadas en adaptabilidad o de otro modo son balanceadas en cohesión y extremas en adaptabilidad (Olson $e t$ al., 1983).

Dentro de estos cinco tipos de familia; cuatro (flexiblemente dispersa, caóticamente separada, caóticamente conectada y estructuralmente dispersa) obtienen una mayor frecuencia. En el caso del tipo "flexiblemente dispersa", la dimensión "flexible", de funcionamiento viable, se caracteriza por el liderazgo igualitario y democrático, el cual permite cambios y acuerdo en las decisiones; y aunque la disciplina es algo severa se negocian sus consecuencias y las reglas se hacen cumplir con flexibilidad y también pueden cambiar; y se comparten los roles o funciones. 
La dimensión "dispersa", de funcionamiento problemático, identifica una extrema separación emocional, muy poco involucramiento o interacción entre sus miembros, correspondencia afectiva infrecuente entre sus miembros, falta de cercanía paterno filial, falta de lealtad familiar, necesidad y preferencia por espacios separados. La toma de decisiones es independiente, existen intereses desiguales, la recreación se lleva a cabo individualmente. El interés se focaliza fuera de la familia y los amigos personales son vistos a solas. Para el tipo de familia "caóticamente separada", la dimensión "caótica", de funcionamiento problemático, se caracteriza por su liderazgo limitado o ineficaz, siendo las disciplinas muy poco severas, las decisiones parentales son impulsivas, hay falta de claridad en las funciones, con frecuentes cambios en las reglas que se hace cumplir inconsistentemente (Olson et al., 1983).

En la dimensión "separada", de funcionamiento viable, existe separación emocional, la lealtad familiar es ocasional, el involucramiento se acepta, prefiriéndose la distancia personal, algunas veces se demuestra la correspondencia personal, los límites parent. filiales son claros con cierta cercanía entre padres e hijos, se alienta cierta separación emocional, el tiempo individual es importante pero se comparte parte del tiempo, se prefieren los espacios separados compartiendo el espacio familiar, las decisiones se toman individualmente siendo posibles las conjuntas, el interés se focaliza fuera de la familia, los amigos personales raramente son compartidos con la familia, los intereses son distintos, la recreación se lleva a cabo más separada que en forma (Olson et al., 1983).

En el tipo "caóticamente conectada", la dimensión caótica, de funcionamiento problemático, se caracteriza por su liderazgo limitado o ineficaz, siendo las disciplinas muy poco severas, las decisiones parentales son impulsivas, hay falta de claridad en las funciones, con frecuentes cambios en las reglas que se hace cumplir inconsistentemente.

En la dimensión "conectada", de funcionamiento viable, existe cercanía emocional, la lealtad familiar es esperada, se enfatiza el involucramiento pero se permite la distancia personal. Las interacciones afectivas son alentadas y preferidas, los límites entre los subsistemas son claros con cercanía patern. filial. La necesidad de separación es respetada pero poco valorada, el tiempo que se pasa juntos es importante, el espacio privado es respetado, se prefieren las decisiones conjuntas, el interés se focaliza dentro de la familia, los amigos individuales se comparten con la familia, se prefieren los intereses comunes y se prefiere la recreación compartida que la individual (Olson et al., 1983).

En cuanto al tipo "estructuralmente dispersa", en la dimensión "estructurada", de funcionamiento viable, el liderazgo es autoritario, siendo algunas veces igualitario, la disciplina rara vez es severa, siendo predecible sus consecuencias, es un tanto democrática, los padres toman las decisiones, las funciones son estables pero pueden compartirse, las reglas se hacen cumplir firmemente, pocas son las que se cambian.

La dimensión "dispersa", de funcionamiento problemático, identifica una extrema separación emocional, muy poco involucramiento o interacción entre sus miembros, correspondencia afectiva infrecuente entre sus miembros, falta de cercanía paterno filial, falta de lealtad familiar, necesidad y preferencia por espacios separados, toma de decisiones independiente, existen intereses desiguales, la recreación se lleva a cabo 
individualmente. El interés se focaliza fuera de la familia, los amigos personales son vistos a solas (Olson et al., 1983).

Si se toma en cuenta, la apreciación que tienen los sujetos hacia el funcionamiento de su sistema familiar, en los grados balanceados de cada tipo de familia, se encuentra pues que el liderazgo, la disciplina y las decisiones de tipo democráticas, los cambios en las reglas para contribuir con la adecuada dinámica familiar, la disciplina algo severa pero negociable en sus consecuencias, además del cambio, los roles o funciones dentro de la familia, la lealtad familiar, las decisiones conjuntas son todos aspectos contemplados dentro del nivel convencional del juicio moral, donde el razonamiento se dirige en la conciencia de ser miembro de un grupo social, en la identificación del sujeto con las normas de su grupo y reconocer que la sociedad espera que actúe de acuerdo a las normas establecidas, respondiendo a las expectativas sociales (Olson et al., 1983).

Como se ha podido apreciar en los resultados, el perfil del nivel de juicio moral de la muestra predomina ampliamente en el nivel convencional, dentro del cual el mayor número de sujetos se sitúa en el Estadio III, de manera similar a la investigación de Romo (2004) y la de Barba y Romo (2005).

A pesar de que la muestra estuvo compuesta por estudiantes universitarios, no se encuentra ningún resultado dentro del nivel post convencional, tal como sucedió en la investigación de Barba (2002), donde se encontró que los sujetos con un mayor nivel de educación y mayor edad, avanzaban progresivamente en el nivel de juicio moral. Sin embargo, es necesario notar que la muestra de dicha investigación, contó con un mayor rango de edad donde se pudo apreciar el desarrollo etápico del juicio moral.

El hecho de que no se hayan encontrado resultados más elevados del nivel de juicio moral en la presente investigación (a pesar de que la muestra cuenta con grado de instrucción superior) apoya el postulado que indica que el desarrollo de los periodos cognitivos son una condición necesaria pero no suficiente para el desarrollo de los niveles paralelos del juicio moral (Kohlberg, 1992); por ello, la asociación significativa encontrada, entre el nivel del juicio moral convencional y el nivel de funcionamiento familiar de rango medio, apoya el hecho de que una dinámica familiar saludable se concentra en un mayor número con un razonamiento moral de segundo nivel. Aquello también podría señalar que la familia juega un rol importante en el desarrollo del juicio moral, puesto que es la institución encargada de la enseñanza de los roles básicos, así como el valor de las instituciones sociales y los modos de comportarse propios de la sociedad (Dugui et al., 1995). Además, tal como lo indicó Kohlberg (1998), la adopción de roles, así como también el afecto y la comprensión de las emociones, están presentes en el desarrollo de las secuencias o estadios del juicio moral; aspectos que están presente dentro de la dinámica familiar según el Modelo Circumplejo (Olson et al., 1983).

\section{CONCLUSIONES}

1. El nivel de juicio moral predominante de la muestra, se encuentra dentro del nivel convencional. 
2. El nivel de funcionamiento familiar predominante en la muestra es la "de rango medio".

3. Existe una relación estadísticamente significativa entre el nivel de funcionamiento familiar de rango medio y el nivel convencional de juicio moral.

4. Tal como lo describe Kohlberg (1992), la muestra se agrupó en su mayor cantidad dentro del nivel convencional de juicio moral.

\section{REFERENCIAS BIBLIOGRÁFICAS}

1. Alarcón, R. (2008). Métodos y diseños de investigación del comportamiento. Lima: Editorial Universitaria URP.

2. Barba, B. y Romo, J. (2005). Desarrollo del juicio moral en la educación superior. En: Revista Mexicana de Investigación Educativa, 24(10), 67-92.

3. Dughi, P., Macher, E., Mendoza, A. y Núñez, C. (1995). Salud mental, infancia, y familia. Lima: UNICEF.

4. Kohlberg, L. (1992). Psicología del desarrollo moral. Bilbao: Desclée de Brouwer.

5. Kohlberg, L., Power, F. y Higgins, A. (1998). La educación moral según Lawrence Kohlberg. Barcelona: Gedisa.

6. Lafosse, S. (1984). Crisis familiar y crisis social en el Perú. En: Revista de la Pontificia Universidad Católica del Perú, 5, 99-112.

7. Minuchin, S. y Fishman, H. (1981). Técnicas de terapia familiar. Barcelona: Paidós.

8. Olson, D. (1985). FACES III (Family Adaptation and Cohesion Scales). St. Paul: University of Minnesota.

9. Olson, D., Russell, C. y Sprenkle, D. (1983). Circumplex model of marital and family systems: VI Theoretical update. Family Process, 22, 69-83.

10. Piaget, J. (1987). El criterio moral en el niño. Barcelona: Martínez Roca.

11. Polain.Lorente, A. y Martínez, P. (1998). Evaluación psicológica y psicopatológica de la familia. Madrid: Rialp.

12. Rokeach, M. (1973). La naturaleza de los valores humanos. New York: La Prensa Libre.

13. Sellman, R. (1971). Taking another's perspective: Role taking development in early childhood. Child Development, 42,121-134.

14. Shaffer, D. (2000). Psicología del desarrollo. Infancia y adolescencia. México: Thomson learning.

15. Siegel, S. y Castellan, N. (1995). Estadística no paramétrica: Aplicada a las ciencias de la conducta. México: Editorial Trillas. 